\title{
A New Method of Machine Tool Thermal Error Modeling Based on the Finite Element Analysis
}

\author{
Jiliang Zhang ${ }^{1, \text { a }}$, Weiguo Gao ${ }^{1, b^{*}}$, Dawei Zhang ${ }^{1}$, Weizhan Zhang ${ }^{1}$, \\ Wenfen Chang ${ }^{1,2}$ and Yingxin $\mathrm{Nie}^{1,2}$ \\ ${ }^{1}$ Tianjin Key Laboratory of Equipment Design and Manufacturing Technology, Tianjin University, \\ Tianjin 300072, China \\ ${ }^{2}$ Beijing Precision Machinery\& Engineering Research Co., Ltd., Beijing 101312, China \\ ajiliang_zhang@tju.edu.cn, ${ }^{b^{*}}$ Corresponding Author: gaowg@tju.edu.cn
}

Keywords: Thermal error, Error model, Finite element analysis.

\begin{abstract}
In this paper, a new method was proposed to detect and model the thermal error of machine tools. This method was aimed at two mutually perpendicular direction deformation, and easily detectable deformation was used to fit the deformation in another direction. In order to verify the correctness of the method, a machine center was analyzed at different thermal conditions with finite element analysis method. Furthermore, the thermal error model of the guide rail on the column was established. The simulation results showed that a reasonable mathematical model for thermal error can be established with this method.
\end{abstract}

\section{Introduction}

The internal heat, generated from the running machine tool, gives rise to its unexpected temperature fluctuation, and consequently results in the machine thermal errors [1]. Thermal effects can contribute more than $50 \%$ to the overall error [2]. There are two ways to improve the accuracy of machine tools: (1) design and manufacture for precision and (2) error compensation [3]. The compensation method is a gradation approach which can manufacture higher accuracy products with lower accuracy machine tools [4].

Reasonable thermal error model can improve the compensation performance. Various techniques have been employed in the modeling of thermal errors in machine tools. Most frequently the principle of regression has been applied in predicting the error where the error is defined as a function of the temperature of critical elements on the machine [5]. In this paper, a new model method, which was defined as a function of the perpendicular direction deformation, was presented. Then, to verify the correctness of the method, a cantilever beam model was analyzed with the finite element analysis method. Finally, a machine center was analyzed at different thermal conditions. And the thermal deformation model of the above guide rail on the column was established to verify the correctness of the method.

\section{The Principle of the Thermal Error Modeling Method}

In view of the machine tool thermal error detection and modeling, this paper put forward a new method. The specific principle was shown in Fig. 1. The right side of the model was fixed. After the model was heated, bending was likely to result in the following deformation as the figure shown. At this time, the two orange lines would have different deformations. Through analysis the Z-direction thermal deformation, the error model in Y-direction could be successfully established. 


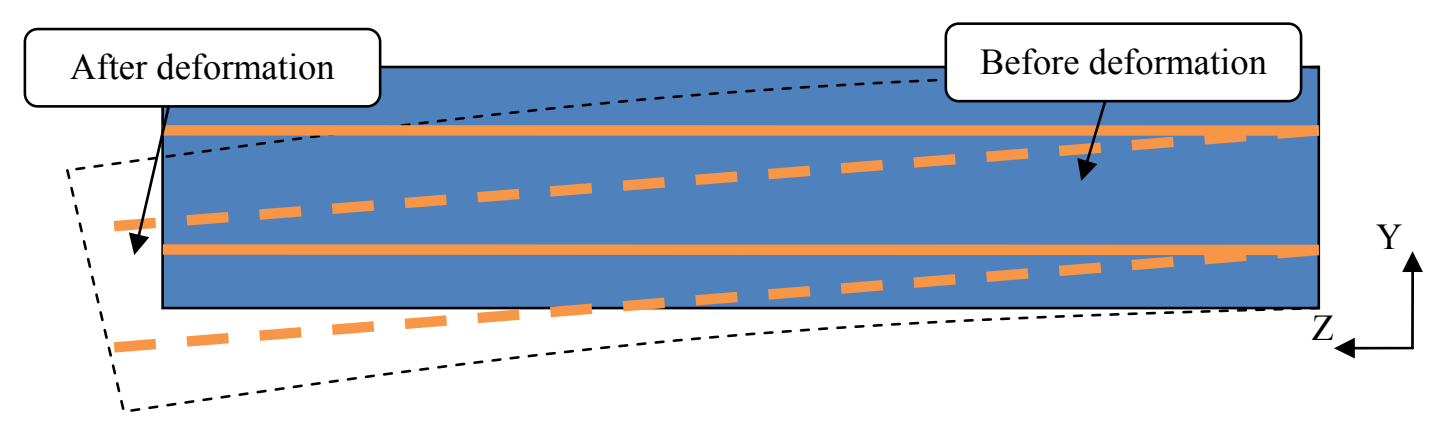

Fig. 1 The principle of the thermal error detection method

\section{The finite element analysis of a cantilever beam model}

To verify the correctness of the method, a cantilever beam model was analyzed with Ansys. Three points were taken at the end surface of the cantilever beam, and their Z-direction deformations were used to fit the deformation in Y-direction.

Material attributes was set according to the nature of QT500-7. Ambient temperature was set to $20^{\circ} \mathrm{C}$. Acceleration of gravity was taken as $9.8 \mathrm{~m} / \mathrm{s}^{2}$. The convection coefficient was defined as 10 $\mathrm{W} /\left(\mathrm{m}^{2} \cdot \mathrm{K}\right)$. The $\mathrm{X}, \mathrm{Y}$ and $\mathrm{Z}$ DOFs of the right side were constrained. Temperature loads were applied on the top surface. After these settings, the mesh model was as shown as Fig. 2.

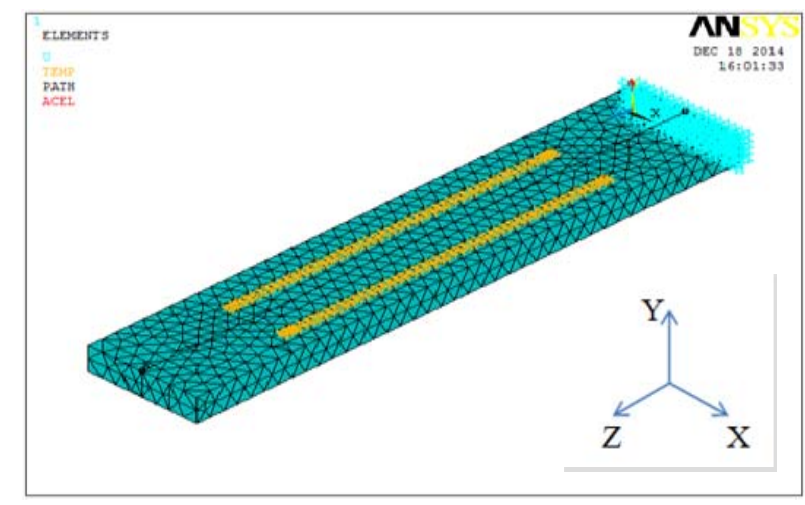

Fig. 2 FEM mesh model

Establish the thermal deformation model. The performance of the model was estimated through structure-thermal analysis under different conditions. Limited to the space, two results were recorded in Fig. 3. 


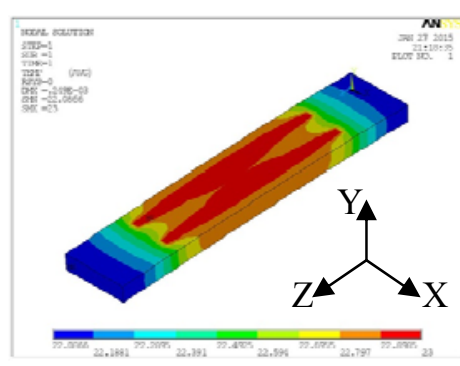

a) Temperature distribution

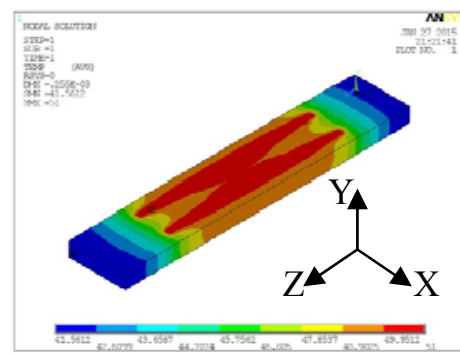

d) Temperature distribution

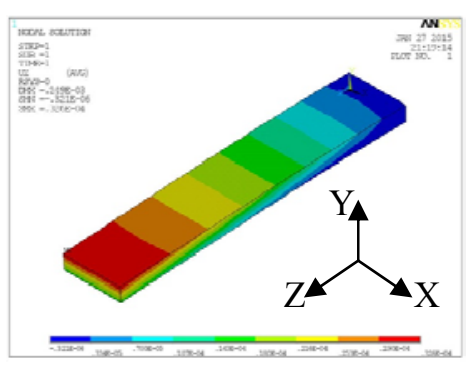

b) Z-direcion deformation

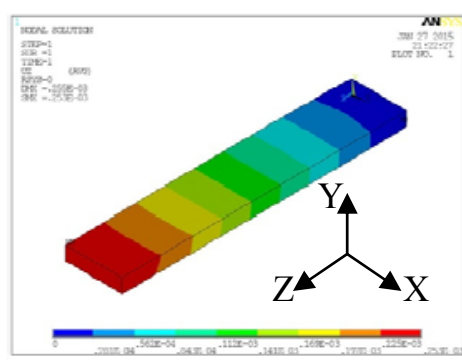

e) Z-direcion deformation

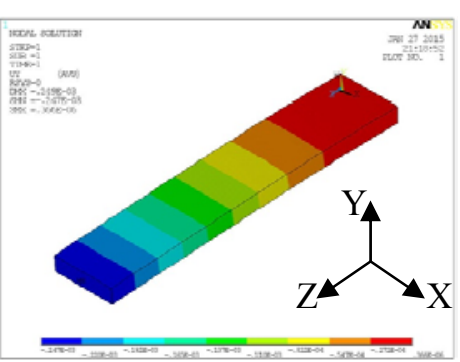

c) Y-direcion deformation

Fig.3 The finite elements analysis results of the model

a)-c) the results of the model under $23^{\circ} \mathrm{C}$ temperature load; d)-f) the results of the $51{ }^{\circ} \mathrm{C}$ temperature load

As it can be seen from the results of the finite element analysis, the temperature distribution and the deformation presented similar laws. Through analysis of these results, polynomials with the same order were used to fit the deformation of the model under different temperature loads. And by comparing the coefficients of the polynomials, a compound expression was established eventually, which was shown as Eq. 1.

$$
\delta_{y}=\boldsymbol{p} \cdot \boldsymbol{z} .
$$

where $\boldsymbol{p}=\left(\begin{array}{lll}p_{1} & p_{2} & p_{3}\end{array}\right), \boldsymbol{z}=\left(\begin{array}{lll}z^{3} & z^{2} & z\end{array}\right)^{\mathrm{T}}, \delta_{y}$ meant the Y-direction deformation of the model midline. $z$ was defined as coordinate value. And $p_{\mathrm{i}}$ were on behalf of the coefficients of the polynomial. At the same time, $\boldsymbol{p}$ was also defined as function. It can be expressed as Eq. 2.

$$
\boldsymbol{p}=\mathbf{A} \cdot \boldsymbol{\delta}_{z}+\boldsymbol{B}
$$

where $\boldsymbol{\delta}_{z}=\left(\begin{array}{ccc}\delta_{z 1} & \delta_{z 2} & \delta_{z 3}\end{array}\right)^{\mathrm{T}}, \delta_{z i}$ were the Z-direction deformation of the three points that were taken at before. A was $3 \times 3$ coefficient matrix. And $\boldsymbol{B}$ was defined as $3 \times 1$ constant vector.

Verify the correctness. In the above, the thermal deformation model of the cantilever beam had been established. To verify the correctness of the model, the simulation results were recorded when the cantilever beam model was applied $23^{\circ} \mathrm{C}, 41^{\circ} \mathrm{C}$ or $51^{\circ} \mathrm{C}$ temperature load. The Z-direction deformation data of the three points were taken into Eq. $1 \sim$ Eq. 2 to get the predicted deformation of the cantilever beam. And the predicted deformation was compared with the result that extracted from Ansys directly. The comparative results were as the Fig. 4 shown. 


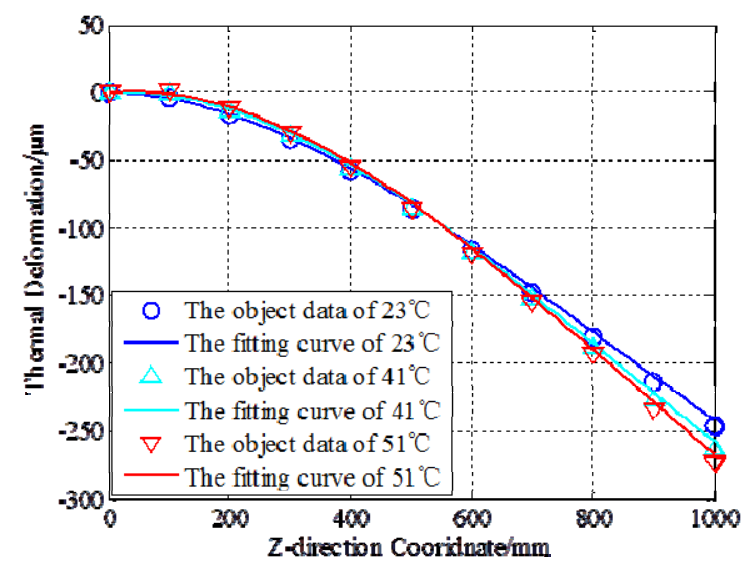

Fig. 4 The comparative results

The figure showed the deviations of the predicted results and simulation results were little. So this method was feasible to establish the thermal deformation model.

\section{The finite element analysis of a machine center}

Analogy to the above research methods, a machine center was analyzed with Ansys under different conditions. The CAD model was shown as below.

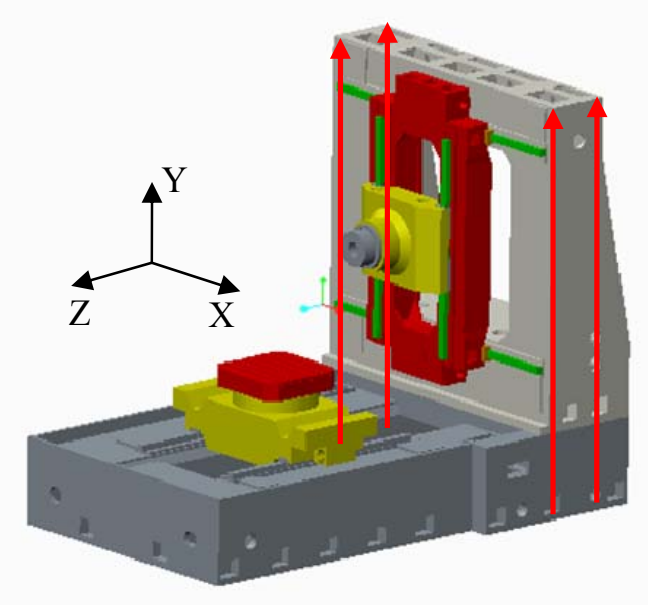

Fig. 5 The CAD model of the machine center

where the red lines' deformations in Y-direction were taken out to fit the Z-direction deformation of the column. The material attributes were set according to the nature of QT500-7 and 45 steel. Refer to the related literature [6], the thermal loads and thermal resistances were set up. And The X, Y and $\mathrm{Z}$ DOFs of the bottom surface were constrained. The FEM mesh model was shown as below. 


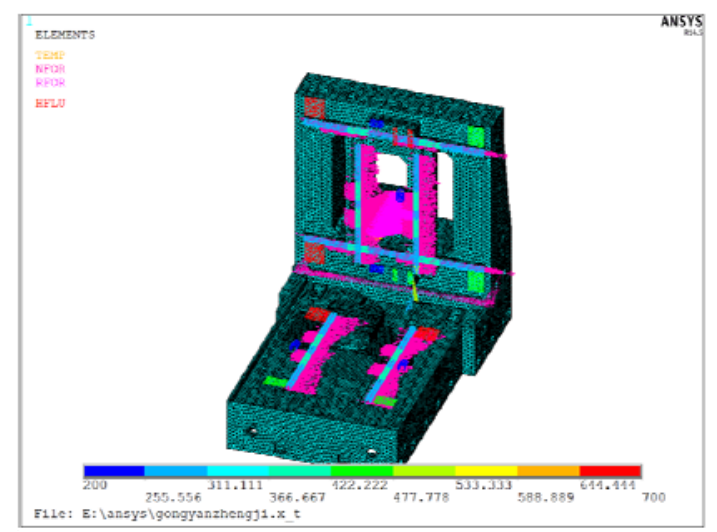

a) Thermal loads

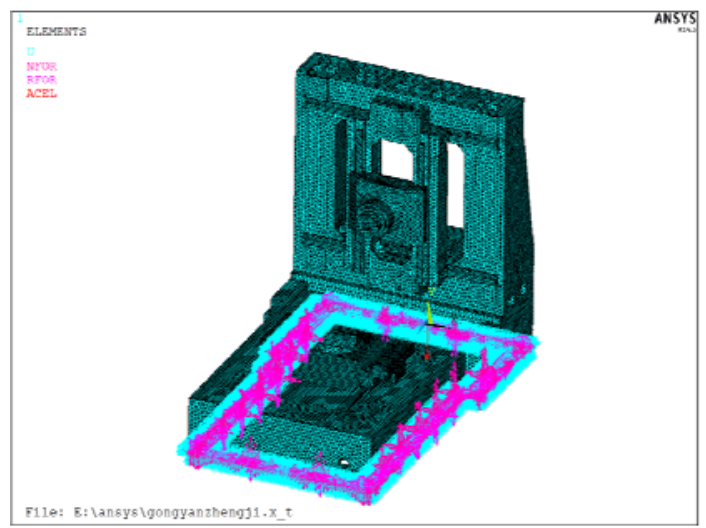

b) The constraint of the bottom surface

Fig. 6 The FEM mesh model

Structure-thermal analysis of the machine center. In accordance with the above settings, the machine center was estimated with the structure-thermal analysis method, under different loading conditions.

Establish the thermal deformation model of the above guide rail on the column. Through analysis the simulation results, second-order polynomials were used to establish thermal deformation model of the above guide rail. And Y-direction deformations of red lines, shown in Fig. 5 , were used to fit coefficients of the polynomial. So the compound expression of the above guide rail of the column can be gained.

$$
\delta_{z}=\boldsymbol{p} \cdot \boldsymbol{x}
$$

In Eq. 3, $\boldsymbol{p}=\left(\begin{array}{lll}p_{1} & p_{2} & p_{3}\end{array}\right), \boldsymbol{x}=\left(\begin{array}{lll}x^{2} & x & 1\end{array}\right)^{\mathrm{T}}, \delta_{z}$ meant the Z-direction deformation of the above guide rail of the column. $x$ was coordinate value. And $\boldsymbol{p}$ was coefficient vector of the polynomial. It was also defined as function.

$$
\boldsymbol{p}=\mathbf{A} \cdot \boldsymbol{y}+\boldsymbol{B} \text {. }
$$

where $\boldsymbol{y}=\left(\begin{array}{llll}y_{1} & y_{2} & y_{3} & y_{4}\end{array}\right)^{\mathrm{T}}$. And $y_{i}$ were on behalf of the Y-direction deformations of red lines. A was $4 \times 4$ coefficient matrix. $B$ was the constant vector.

Verify the correctness. Two simulation results were used to verify the correctness of the thermal deformation model. In order to get the predicted deformation, the Y-direction deformation of the red lines were taken into the Eq. 3 Eq.4. The predicted deformations were compared with the simulation results, which were extracted from Ansys directly. The fitting effective was shown as below.

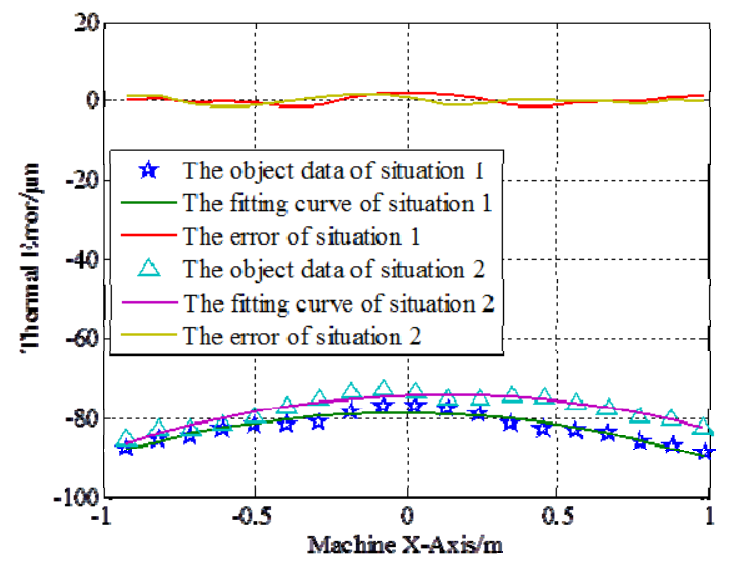

Fig. 7 The comparative results 


\section{Conclusions}

A new model method, which was defined as a function of the perpendicular direction deformation, was presented in this paper. Through analysis of the cantilever beam model, the method was proved to be feasible to establish the thermal error model. And to verify the effective of the method, a machine center was estimated with Ansys under different conditions. Furthermore, the method was used to establish the thermal deformation of the above guide rail of the column. The fitting results showed that this method can be helpful to model about thermal error.

\section{Acknowledgements}

This paper is supported by the state S\&T projects for upmarket NC machine and fundamental manufacturing equipments of China (No. 2014ZX04014-011).

\section{References}

[1] Liu, T.; Gao, W.; Tian, Y. et al. A differentiated multi-loops bath recirculation system for precision machine tools. Applied Thermal Engineering, 2015, 76: 54-63.

[2] Week M.; Mckeown P.. Reduction and compensation of thermal error in machine tools. CIRP Annals Manufacturing Technology, 1995, 44(7): 589-598.

[3] Tian, W.; Gao, W.; Zhang, D. et al. A general approach for error modeling of machine tools. International Journal of Machine Tools \& Manufacture, 2014, 79: 17-23.

[4] Cui, G.; Lu, Y.; Gao, D. et al. A novel error compensation implementing strategy and realizing on Siemens 840D CNC systems. International Journal of Advanced Manufacturing Technology, 2012, 61(5-8): 595-608.

[5] Ramesh, R.; Mannan, M.; Poo, A. et al. Thermal error measurement and modeling in machine tools. Part II. Hybrid Bayesian Network--support vector machine model. International Journal of Machine Tools and Manufacture. 2003, 43(4): 405-419.

[6] Zhang D.; Yan J.; Gao W. et al. Structural thermal error modeling of machine tools in working space based on CAE thermal analysis. Journal of Tianjin University. 2015, doi: 10.11784/tdxbz201501079. 